\title{
A Meta-Analysis of the Effectiveness of Kanglaite Injection Plus First-Line EGFRTKIs Versus First-Line EGFRTKIs Alone Stage IIIB Lung Adenocarcinoma
}

\author{
David Kayembe Mwimbi ${ }^{1}$, Zhang Gaochenxi ${ }^{1}$, Wenpei Zhu ${ }^{1}$, Ying Wang ${ }^{1}$, Liang $\mathbf{Y i}^{2}$, Wang $\operatorname{Dan}^{3}$, \\ Chen Huihui ${ }^{4}$, Shu Qijin ${ }^{4,}$ " \\ ${ }^{1}$ The First Clinical Medical College of Medicine, Zhejiang Chinese Medical University (International Education College IEC), Hangzhou, P. R. \\ China \\ ${ }^{2}$ The Third Affiliated Hospital of Zhejiang Chinese Medical University, Hangzhou, P. R. China \\ ${ }^{3}$ The First Affiliated Hospital, College of Medicine, Zhejiang University, Hangzhou, P. R. China \\ ${ }^{4}$ Department of Medical Oncology, The First Affiliated Hospital of Zhejiang Chinese Medical University, Hangzhou, P. R. China
}

Email address:

shuqihz@163.com (Shu Qijin)

*Corresponding author

\section{To cite this article:}

David Kayembe Mwimbi, Zhang Gaochenxi, Wenpei Zhu, Ying Wang, Liang Yi, Wang Dan, Chen Huihui, Shu Qijin. A Meta-Analysis of the Effectiveness of Kanglaite Injection Plus First-Line EGFRTKIs Versus First-Line EGFRTKIs Alone Stage IIIB Lung Adenocarcinoma. International Journal of Clinical Oncology and Cancer Research. Vol. 5, No. 3, 2020, pp. 43-55. doi: 10.11648/j.ijcocr.20200503.11

Received: February 27, 2020; Accepted: March 12, 2020; Published: July 23, 2020

\begin{abstract}
Objective: Several systematic reviews for Therapeutic Effect of Kanglaite Injection Plus First-Line Epidermal Growth Factor Receptor (EGFR) Tyrosine Kinase Inhibitors (TKIs) Versus First-Line Epidermal Growth Factor Receptor (EGFR) Tyrosine Kinase Inhibitors (TKIs) Alone In Stage IIIB Advanced Lung Adenocarcinoma have recently emerged evidence. However, limited data are available regarding the activity of available EGFR TKIs against uncommon EGFR mutations. This meta-analysis evaluates the efficacy of Kanglaite Injection Plus First-Line Epidermal Growth Factor Receptor (EGFR) Tyrosine Kinase Inhibitors (TKIs) Versus First-Line Epidermal Growth Factor Receptor (EGFR) Tyrosine Kinase Inhibitors (TKIs) Alone In Stage IIIB Advanced Lung Adenocarcinoma. So therefore, Lung Adenocarcinoma related diseases have a profound economic impact on health care systems global wide, thus Kanglaite Injection combined with First-Line EGFR -TKIs have been shown to have beneficial effects than treatment with First-Line EGFR Tyrosine Kinase Inhibitors (TKIs) Alone. Methods: We electronically searched the literature of the China National Knowledge Infrastructure (Chinese language, English 2010-2019), Pub Med, Cochrane Central Register of Controlled Trails from database inception, CNKI, web of science Wang Fang, and manually searched Chinese-language oncology journals to identify randomized controlled trials (RCTs) of Kanglaite Injection Plus First Line EGFR-TKIs Versus First Line EGFR- TKIs Alone, regardless of their having been published or not, blinding, duration of treatment, or duration of follow-up. The quality of the included trials was assessed using the method recommended by The Cochrane Collaboration (CC). If heterogeneity existed among subgroups, then overall results (OS) were calculated based on a random-effects model; otherwise, a fixed effects model was used. Results: Electronic database searches yielded 1780 citations with NSCLC. Articles or Records Excluded by screening of the title/abstract level total 510, Records which are not Rcts 430, Study with no EGFR mutation analysis 590, Due to duplicated publication 180. Finally, we identified full text articles retrieved for detailled evaluation 70. The sample size of each trial had calculated by Rev Man 5.3. Pooled analyses performed using both fixed- and random-effects models revealed that compared with First Line of Egfr-Tkis alone, KLT injection plus First Line of Egfr-Tkis improved the response rate (relative risk [RR \}, 1.34; 95\% CI, 1.19-1.51 and RR, 1.35; 95\% CI, 1.2 0-1.51, respectively). KLT injection plus First Line of Egfr-Tkis was associated with improvement in the symptoms of cough, dyspnea, chest pain, fatigue, and anorexia.
\end{abstract}

Keywords: Meta-Analysis, Kanglaite Injection, Egfr-Tkis, Stage IIIB A Lung Adenocarcinoma 


\section{Introduction}

Populations who are suffering from Cancer have a higher likelihood of serious health complications [1]. Data from the latest global cancer statistics show that there will be 18.1 million new cancer cases and 9.6 million cancer deaths in 2018. Therefore, Lungs Cancer Also called Bronchogenic carcinoma with its main types of Non-Small Cell Lungs Cancer (NSCLC 80-85\%) \& Small Cell Lungs Cancer (SCLC $15-20 \%$ ) is most a serious threat to human health and life see figure 1. It is also the major cause of cancer-related deaths in the Western world [2], leading to about $80-85 \%$ of all lung cancer cases. NSCLC is characterized by being a highly heterogeneous disease, harboring many genetic aberrations within the various subtypes [3]. More than half of the NSCLC cases are diagnosed at an advanced stage (stages III and IV) [2], and $63 \%$ of cases are 65years of age or older. Demographics that are shifting toward an older population suggest that oncologists will be seeing more elderly patients with NSCLC in years to come. In patients with NSCLC, only $20-25 \%$ of the cases harbor treatable drive mutations, such as Epidermal Growth Factor Receptor (EGFR) mutation, for which tyrosine kinase inhibitor can be used. $75 \%-80 \%$ of NSCLC cases are EGFR wild type and cannot gain any benefit from target therapy [3]. Bevacizumab, a monoclonal antibody which targets vascular endothelial growth factor (VEGF), can be applied to EGFRwild-type NSCLC, but does not improve overall survival (OS) for patients over the age of 65 . To date, Egfr-Tkis remains the standard treatment of care In Stage IIIB-IV adenocarcinoma lung cancer with EGFR mutation-positive for older patients with good functional status. The development of first-, second-, and third-generation epidermal growth factor receptor (EGFR) Tyrosine kinase inhibitors (TKIs) has revolutionized the Treatment of patients with non-small cell lung cancer (NSCLC) harboring mutations in the EGFR. However, limited data are available regarding the activity of available EGFR TKIs against uncommon EGFR mutations.

Furthermore, epidermal growth factor receptor-tyrosine kinase inhibitors (EGFR-TKIs) have shown excellent efficacy in advanced EGFR-mutation positive NSCLC, with almost $70 \%$ of response rate. Several randomized controlled trials (RCTs) have confirmed that first-line EGFR-TKI regimen can improve tumor response rate and progression-free survival (PFS) in patients with advanced EGFR-mutant NSCLC. But it still remains appealing to explore whether EGFR-TKI can be applied to neoadjuvant/adjuvant therapy for resectable stages [4], in order to improve DFS and overall survival (OS). Upon EGFR-TKIs appearing on the market, some surgeons began recommending TKIs during perioperative period. However, since applications of TKI perioperatively are associated with novel concepts in translational researches, it still remains a highly controversial issue up to now. Different biological characteristics between early and advanced lung cancer can be detected in previous reports and proved by several phenomena. For example, high levels of serum tumor markers, such as carcinoembryonic antigen (CEA), cytokeratin19 fragment antigen 21-1 (CYFRA21-1) and cancer antigen 125 (CA125), are negative prognostic factors in early-stage NSCLC but positive prognostic factors in advanced NSCLC; early stage tumors are more mobile than locally advanced stage NSCLC, which need to be integrated into precise radiotherapy planning [5].

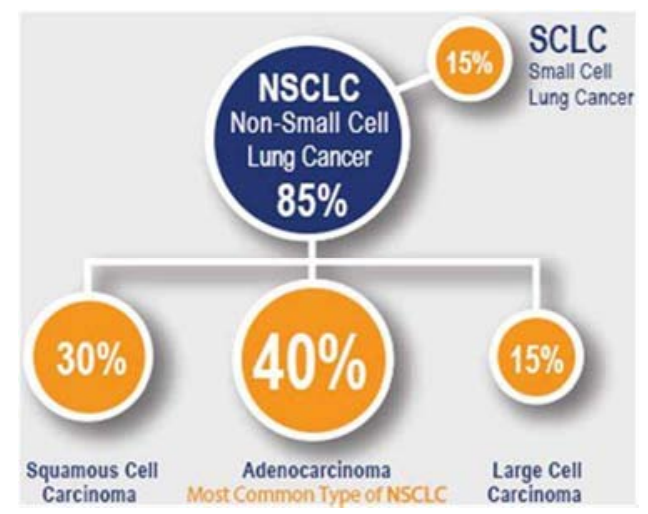

Figure 1. Main types of (NSCLC 80-85\%) \& (SCLC 15-20\%).

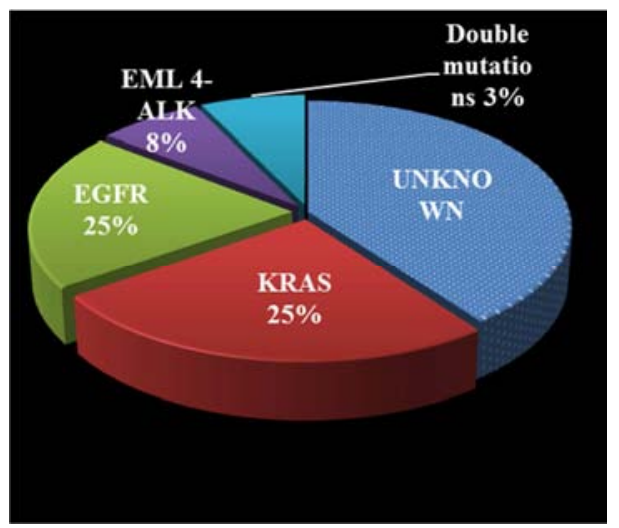

Figure 2. Incidence of mutation-status in NSCLC.

\subsection{Study Objectives}

The aim of this Article is to evaluate the Effect of Kanglaite Injection plus First-Line Epidermal Growth Factor Receptor (EGFR) Tyrosine Kinase Inhibitors (TKIs) Versus First-Line Epidermal Growth Factor Receptor (EGFR) Tyrosine Kinase Inhibitors (TKIs) Alone in Stage IIIB Advanced Lung Adenocarcinoma.

\subsection{Epidemiology \& Statistic}

Lung cancer is the most common cause of death from cancer worldwide, responsible for nearly one fifth of all cancer deaths (1.59 million deaths, $19.4 \%$ of the total). It is also the most commonly diagnosed and leading cause of death by cancer in men in the United States according for $27 \%$ and $31 \%$ of all cancer death in Women and men, respectively. The median age of diagnosis is 70 years. Although many patients achieve disease-free survival (DFS), some experience a long-term impairment of their quality of life, and disease recurrence is common [6]. Therefore three surveys found that the prevalence of EGFR mutations in NSCLC patients was 
approximately $10 \%$ in Europe, $17 \%$ in the United States, and $78.8 \%$ in East Asia. The PIONEER study in 2014 was the first to confirm a high frequency of EGFR mutations $(51.4 \%)$ in Asian patients with lung adenocarcinoma, with a rate of $62.1 \%$ in Taiwan. An epidemiological study in 2015 reported that the EGFR mutation rate among patients with treatment-naïve lung adenocarcinoma in Taiwan was $55.4 \%$, with the main mutations being del 19 (44.8\%) and L858R point mutations (47.9\%) [6].

In 1974 in the UK $51 \%$ of men and $41 \%$ of Women smoked cigarette-nearly half the adult population. Now, about $21 \%$ of men and $19 \%$ of women aged 16 years and over smoke. The highest rates are in people aged $20-24$ (28\% of women and $30 \%$ of men in this age group smoke). The highest rates of cigarette consumption per capita are in Serbia, Bulgaria, Greece, China and Russia. In Global terms, the USA ranks 51st and the UK ranks 73rd, close to the rates in Sweden, Canada and the Netherlands.

Lung cancer imposes a considerable public health and economic burden in Asian countries. In mainland China, for example, the incidence of lung cancer has increased rapidly over the past 2 decades, especially in females. In 2005, there were 497,908 new cases and 428,936 deaths in China, the highest values for any malignancy. Ten-year relative survival for lung cancer from the Singapore Cancer Registry was 5.2\% in men and 7.2\% in women for the years 1998-2002, with only pancreas and liver cancers having similar or lower survival rates. Smoking rates and habits have a primary influence on lung cancer incidence in Asian countries, and smoking rates are ominously high, particularly among Asian men. Approximately two-thirds of adult Chinese men are smokers, and with smoking rates increasing and yet to peak in China and other developing countries, deaths due to lung cancer are expected to reflect this situation in the future. However, risk factors for Non-Small lung cancer vary among local population groups and can include ambient urban air pollution as well as occupational exposures such as cooking fumes. Finally, despite the importance of smoking and environmental pollutants, it is notable that never smokers with non-small cell lung cancer are found disproportionately high in Asian patients, particularly females with adenocarcinoma.

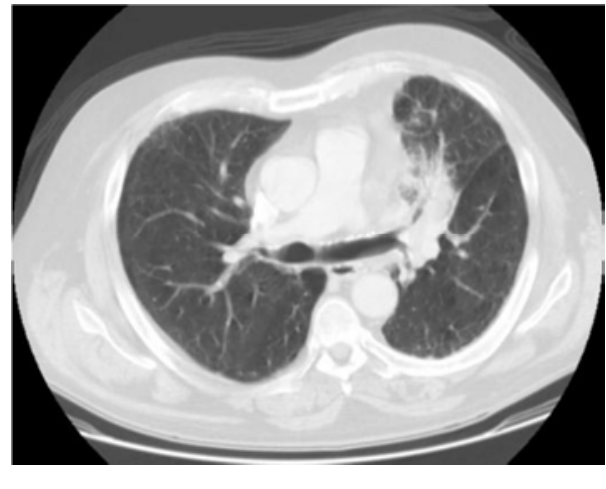

Figure 3. Chest X-ray.

\section{Study Design and Treatment}

Pathologically, there are 100 types of cancer, including lung cancer, breast cancer, skin cancer or Kaposi sarcomas, colon cancer, prostate cancer, and lymphoma. Cancer starts when a cell is somehow altered so that it multiplies out of control. A tumor is a mass composed of a cluster of such abnormal cells. Most cancers form tumors, but not all tumors are cancerous. Researchers are attempting to find screening tests that may help to dignose lung cancer early, such as Sputum cytology, CT, Imaging tests, and tissue samples (Biopsy) see table 1 [7].

SCLC is more responsive to Chemotherapy than NSCLC. While NSCLC accounts for $80 \%$, including three subtypes: Adenocarcinoma that is the most common form of lung cancer in the United States among both men and women; Squamous Cell Carcinoma, which accounts for about $25 \%$ of all lung cancers; Large Cell Carcinoma, which accounts for about $10 \%$ of NSCLC tumors. Each subtype relates to the specific type of cell affected and are grouped together because they behave in a similar way. Furthermore, staging lung cancer is based on whether the cancer is local or has spread from the lungs to the lymph nodes or other organs. Because the lungs are large, tumors can grow in them for a long time before they are found. Even when symptoms - such as coughing and fatigue- do occur, people think they are due to other causes. For this reasons, early-stage lung cancer (stage I \& II) is difficult to detect. Staging in Small Cell Lung Cancer, as limited stage, in this stage, cancer is found on one side of the Chest, involving just one part of the lung and nearby lymph nodes. Extensive stage, in this stage, cancer has spread to other regions of the Chest or other parts of the body. While, stages of Non-Small Cell Lung Cancer as known, stage I, the cancer is located only in the lungs and has not spread to any lymph nodes. Stage II, cancer is in the lung and nearby lymph nodes. Stage III, Cancer is found in the lung and in the lymph nodes in the middle of the Chest, also described as locally advanced disease. Stage III which has two subtypes, if the Cancer has spread only to lymph nodes on the same side of the Chest where the cancer started, it is called stage IIIA. If the Cancer has spread to the lymph nodes on the opposite side of the Chest, or above the collar bone, it is called stage IIIB. While Stage IV, this is the most advanced stage of lung cancer, and therefore is also described as advanced disease. This is when the cancer has spread to both lungs, to fluid in the area around the lungs, or to another part of the body, such as the liver or other organs [8]. The management of lung cancer should primarily be based on the histopathological type, secondarily on the stage. NSCLC represents one treatment group and SCLC another. Accordingly, the treatment will be described separately. Less common lung tumors include neuroendocrine tumors including carcinoids and pleural malignancies, such as mesothelioma. Tyrosine kinase inhibitors (TKIs) are medicines that block signals that tell a cell to grow and divide. This can slow or stop cancer cells from growing. In some cases it can cause the cells to die. Why It Is Used. Tyrosine kinase inhibitors (TKIs) are medicines used to treat many different cancers, such as leukemia and non-small cell lung cancer. 
Table 1. Clinical Drugs \& dosage of EGFER TKIs.

\begin{tabular}{lll}
\hline Generations of EGFR-TKIs & Drugs & Daily dosage \\
\hline \multirow{2}{*}{ First generation Egfr-Tkis } & Erlotinib & $150 \mathrm{mg}$ capsules \\
& Gefitinib & $250 \mathrm{mg}$ capsules \\
\multirow{2}{*}{ Second generation Egfr-Tkis } & Afatinib & $40 \mathrm{mg}$ capsules \\
& Dacomitinib & $125 \mathrm{mg}$ capsules \\
Third generation Egfr-Tkis & Osimertinib & $150 \mathrm{mg}$ capsules \\
\hline
\end{tabular}

Patients assigned to TKIs received oral EGFR-TKI Erlotinib (Roche, Switzerland) $150 \mathrm{mg}$, Gefitinib (AstraZeneca, UK) $250 \mathrm{mg}$, or Icotinib (Icotinib Beta, China) $125 \mathrm{mg}$ per dose, three times per day; the drug was chosen by the patients] plus oral CHM. First generation targeted Egfr 19 del exon and 21 L858R exon mutations. The second generation targeted Egfr 18, 19, 21 exon mutation and ERBB2 amplification. Beyond first-line treatment almost all patients who benefit from EGFR-TKIs will eventually develop clinical resistance. About half of the resistance is explained by the acquired EGFR exon 20 T790M mutations. The third targeted Egfr mutation and 20 T790 M (20 T 790 M exon mutation) while the third can solve the resistance of first and second generation induced by Egfr 20 T790 M exon mutation. This study has established a new paradigm: all patients with clinical resistance to first-/second-generation EGFR-TKIs should be tested for the presence of T790M mutation and Osimertinib should be offered as standard treatment for patients who test positive. Gefitinib (Iressa) approved by the U.S. Food and Drug Administration (FDA) for first-line treatment of advanced EGFR mutation-positive NSCLC with EGFR exon 19 deletions or exon 21 (L858R) substitution mutations as detected by a FDA-approved test (13 July 2015); the European Commission has granted marketing authorisation for Gefitinib for the treatment of adults with locally advanced or metastatic NSCLC with activating mutations of EGFR across all lines of therapy (1 July 2009): many countries in Asia including Japan, Korea, Taiwan, China, India and Thailand approved Gefitinib since 2003 as second or third line after chemotherapy. Later on the drug was approved as first line. India had several generic versions of gefitinib available. Erlotinib (Tarceva) received regular approval as monotherapy for the treatment of patients with locally advanced or metastatic NSCLC after failure of at least one prior chemotherapy regimen November 18, 2004, regardless EGFR mutational status. After approval as maintenance. October 18, 2016, the FDA modified the indication for erlotinib for treatment of NSCLC by limiting its use to patients whose tumors have specific EGFR mutations. The labeling change applies to patients with NSCLC receiving maintenance or second or later line of treatment. These indications will be limited to those patients whose tumors have EGFR exon 19 deletions or exon 21 L858R substitution mutations as detected by a FDA-approved test. The first-line indication previously was limited to patients with EGFR exon 19 deletions or exon 21 substitution mutations. Many countries in Asia including Japan, Korea, Taiwan, China, India and Thailand approved the drug erlotinib since 2004 as second or third line. Later on, the drug was also approved as first line. Afatinib (Giotrif) FDA was initially approved in
2013 for the treatment of patients with metastatic NSCLC whose tumors have EGFR exon 19 deletions or exon 21 (L858R) substitution mutations as detected by a FDA-approved test and in 2016 for metastatic, squamous NSCLC progressing after platinum-based chemotherapy (histology- oriented indication regardless EGFR mutational status). January 12, 2018, the FDA broadened the indication to first-line treatment of patients with metastatic NSCLC whose tumors have non-resistant EGFR mutations as detected by a FDA-approved test. Afatinib is approved in several markets, including the EU, Japan, Taiwan and Canada under the brand name Giotrif, in the US under the brand name Giotrif and in India under the brand name Xovoltib for patients with distinct types of EGFR mutation-positive NSCLC.

\subsection{Chinese Medicine Treatment}

It is one of the most common complementary and alternative medicine therapies for lung cancer in China and others countries in Asia. It has a holistic view of the body. Everything is seen to exist within the continuous circle of nature. When the elements of nature are in balance, life is in harmony, and flourishes, humanity cannot be separated from nature; we are nature, manifested as people. Living in harmony with the world around us is the way to maintain health. If one were to live out of balance with nature, illness or disease (western terms) would develop called the body that is suffering from an imbalance, or disharmony. Every Organ in Chinese Medicine has a partner organ. One is yin, the other yang they work together to keep the body in balance [9]. In addition, the lungs are yin and their yang partner is the large intestine (cm theory). According to the conceptual framework of Chinese Medicine, cancer arises when the body constitution becomes imbalanced, which leads to accumulation of toxins and heat and blood stasis and eventually produces carcinogenic factors. In China, Chinese Medicine is covered by the National Health Insurance Research Database (NHIRD) and is a generally established form of medical treatment. Chinese Medicine treatment under the NHI is prescribed by board-certified Chinese medical physicians according to Chinese Medicine syndrome differentiations. The NHIRD is the only computerized reimbursement databases in the world that stores longitudinal prescription data for both Chinese Medicine and Western medicine. This makes the NHIRD an optimal platform to determine the efficacy of Chinese Medicine in reducing the risks of death and disease progression for patients with advanced lung adenocarcinoma treated with first-line EGFR-TKIs table 1. Due to adverse reactions caused by side effects, Chinese Medicine (CM) is reported as a safe alternative therapy with many roles in improving symptoms, such as reducing cancer-related fatigue, improving gastro intestinal side effects, protecting liver function, and even ameliorating bone marrow suppression [10]. And Chinese Medicine, as an adjunct to the conventional antitumor therapy, may improve overall survival of lung cancer patients. As a result of Chinese Medicine's advantage in reducing side effects and its potential role in prolonging survival, in China many elderly patients with advanced 
NSCLC, always use Chinese medicine.

Table 2. Clinical Drugs \& dosage In Chinese medicine.

\begin{tabular}{lllll}
\hline $\begin{array}{l}\text { Prescription Clinical } \\
\text { Trials 2019 }\end{array}$ & formula & $\begin{array}{l}\text { Daily } \\
\text { Dosage }\end{array}$ & formula & dailydosaage \\
\hline & kuangqi & $15 . \mathrm{g}$ & Beishashen & $15 . \mathrm{g}$ \\
CHM used in clinic by & taizishen & $15 . \mathrm{g}$ & Huanglin & $15 . \mathrm{g}$ \\
Prof Shu Qijin et Al & Yujin & $12 . \mathrm{g}$ & diaochong & $15 . \mathrm{g}$ \\
& chuanshidou & $12 . \mathrm{g}$ & baixianpi & $15 . \mathrm{g}$ \\
& Maidong & $15 . \mathrm{g}$ & baihe & $15 . \mathrm{g}$ \\
\hline
\end{tabular}

Kanglaite Injection KLT.

Systematically, the treatment based on journal which is to identify randomized controlled trials (RCTs) of the Effect of Kanglaite Injection Plus First-Line Epidermal Growth Factor Receptor (EGFR) Tyrosine Kinase Inhibitors (TKIs) Versus First-Line Epidermal Growth Factor Receptor (EGFR) Tyrosine Kinase Inhibitors (TKIs) Alone in Stage IIIB Advanced Lung Adenocarcinoma.

1) $200 \mathrm{~mL}$, slow IV drip, once daily for 14 consecutive days, subsequent courses repeated after a 7-day interval.

2) Subjects will be treated for 4 courses (12 weeks).

Other Name: Coicis Oil injection

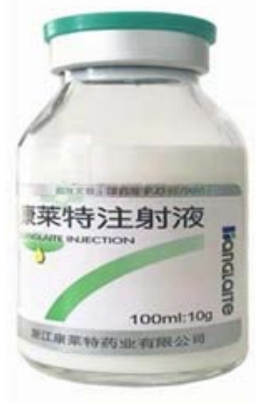

Figure 4. Kanglaite Injection KLT.

Clinically, Kanglaite injection KLT (Zhejiang Kanglaite Group Co. Ltd, Hangzhou, China) is a botanically sourced, molecularly targeted agent that is prepared as a microemulsion for IV use [11]. The active substance is extracted from the herb Semen micis. It is used in Combination with Chemotherapy to minimize toxic reactions and enhance the Chemotherapy. In 1995, KLT Patent certificates were granted in China. In August 1997, Phase III clinical trials were completed and KLT was officially launched in China after final approval from the Ministry of Public Health. Since 1997, > Health500, 000 Health cancer Patients in $>2000$ large- and medium-sized hospitals in China have been treated with KLT. A Semen coicis extract was found to suppress the growth of Squamous lung cancer cells. KLT decreased the number of G21M phase cells, suppressed the proliferation of cancer cells, and induced apoptosis of cancer cells, actions that constitute an important mechanism of the antitumor action of KLT. KLT was found to be effective in reversing multiple-drug resistance of cells and increasing the sensitivity of mouse cancer cells to chemotherapeutic agents [12]. KLT injection might have a direct effect on cancer cell death and improvement of patient's immune function, symptoms, and quality of life. A Cochrane protocol focused on symptom palliation of patients with lung cancer rather than effectiveness. To date, no systematic review or meta-analysis of the effectiveness of KLT injection in patients with primary SCLC has been done.

\subsection{Chinese Medicine Syndrome Differentiation}

Chinese Medicine was chosen from three prescriptions based on TCM syndrome differentiation: a qi-benefiting recipe, a yin-nourishing recipe, and a qi-yin benefiting recipe. One experienced TCM physician was assigned to differentiation syndromes and collected the baseline information. TCM diagnostic data and the prescribed medication for 1 month were given at the first visit. The bioactive components were extracted from water. Some quality control markers were selected by referring to the Chinese Pharmacopoeia (2015 edition), and the quantitative analysis was performed by high-performance liquid chromatography coupled with ultraviolet detection (HPLC-UV) [13].

Three kinds of oral placebos were matched by weight, color, smell, taste, and packaging according to the CHM formulas. The patients took the Chinese medicine applications on the same day as EGFR-TKIs. The CHM granules were dissolved into $150 \mathrm{ml}$ of warm water to drink twice per day after a meal until the end of the EGFR-TKIs treatment. Clinical research pharmacists took part in and supervised the procedures. EGFR-TKI and CHM treatment lasted until disease progression (DP), unacceptable toxicity, or any other study discontinuation criteria were met. According to previous studies on drug-related toxicities (Melosky and Hirsh, 2014; Califano et al., 2015), dose adjustments or delays were implemented. For EGFR-TKIs-treated patients with serious diarrhea, rashes, or any other EGFR-TKIs-related adverse event (AE), the dosage could be stopped for up to 14 days, and appropriate symptomatic treatment could be provided. EGFR-TKIs were stopped once interstitial lung disease occurred. For CHM-treated patients with abnormal liver function, poor appetite, nausea, diarrhea, and other gastrointestinal AEs, CHM could be delayed up to 14 days. TKIs alone, corresponding to a 7-month improvement in PFS based on previous trials using CHM or TKIs alone in the first-line therapy. The PFS of the second-line EGFR-TKIs targeted therapy was 7 months (Ailing Cao 2017), with an inspection level of $\alpha=0.05$ and power $1-\beta=0.80, \mathrm{HR}=0.50$; here, CHM plus EGFR-TKI may increase PFS to 12 months in the second-line therapy of advanced lung adenocarcinoma with EGFR mutation. PFS is illustrated by the Kaplan-Meier survival curve and log-rank test [14]. For the PFS and tumor response, an adjusted Cox regression model was used to estimate the adjusted HRs for differences between the treatment arms with the selected prognostic factors, including the EGFR mutation type, age, sex, EGFR-TKIs drugs, treatment line, smoking status, and ECOG PS. Fisher's exact test was used to compare tumor response rates and the incidence of TEAEs between the arms. $\mathrm{P}<0.05$ was considered statistically significant. Analyses were performed 
using Rev Man version 5.3, SAS version 9.2 (SAS Institute, Cary, NC). Zhejiang Clinical Research Center (ZCRC) experts were invited to manage the statistical analysis.

The results were as follows: calycosin-7-O-beta-D-glucoside $0.012 \%$, icariin $0.075 \%$, psorale isopsoralen $0.067 \%$ in formula I, rosmarinic acid $0.036 \%$, and apigenin $0.0069 \%$ in formula IV, which provided the quality control of the formula. The formulas were applied according to the following regimens (Supplementary Tables 2, 3).

a. Formula I (including tonifying qi and warming yang granules) (Supplementary 1).

b. FormulaII (nourishing yin granules) (Supplementary 2).

Table 3. Traditional Chinese medicine (TCM) syndrome differentiation.

\begin{tabular}{lll}
\hline \multicolumn{2}{l}{ Traditional Chinese medicine (TCM) syndrome differentiation.* } & Tongue diagnosis \\
\hline $\begin{array}{l}\text { TCM syndrome } \\
\text { differentiation }\end{array}$ & Main symptoms & $\begin{array}{l}\text { CHM } \\
\text { regimens }\end{array}$ \\
\hline $\begin{array}{l}\text { 1. Qi syndrome } \\
\text { deficiency }\end{array}$ & $\begin{array}{l}\text { Cough, large amount of sputum, loss of appetite, fatigue and weakness, } \\
\text { pale and bulgy tongue. Secondary symptoms: spontaneous sweat, } \\
\text { unshaped stool, thin superficial, and smooth pulse }\end{array}$ \\
& $\begin{array}{l}\text { Cough, small amount of sputum, dried mouth, red tongue. Secondary } \\
\text { symptoms: night sweats, insomnia, low fever, thready pulse, rapid pulse }\end{array}$ \\
$\begin{array}{l}\text { 2. Yin syndrome } \\
\text { deficiency }\end{array}$ & $\begin{array}{l}\text { Cought, small amount of sputum, dried mouth, red tongue. Secondary } \\
\text { Cough, small amount of sputum, dried mouth, red tongue. Secondary } \\
\text { symptoms: night sweats, insomnia, low fever, thready pulse, rapid pulse }\end{array}$ \\
\hline
\end{tabular}

*Based on the Chinese Medicine New Medicine Clinical Practice Guideline (trial implementation) (published by China Medical Science Press in 2002) and Zhejiang Chinese Medicine Routine Practice (written by the Zhejiang Municipal Commission of Health and Family Planning).

\section{Materials and Methods}

\subsection{Study Design}

This multicenter, randomized, double-blind, placebo-controlled study compared with A Meta-Anal Of The Therapeutic Effect of Kanglaite Injection Plus First-Line Epidermal Growth Factor Receptor (EGFR) Tyrosine Kinase Inhibitors (TKIs) (gefitinib, erlotinib, or icotinib) Versus First-Line Epidermal Growth Factor Receptor (EGFR) (TKIs) Alone as the first- or second-line therapy in Chinese patients with advanced ADC and who had EGFR activating mutations. All of the patients were given informed consent prior to conducting the study. The study procedures and informed consent form were approved by the Institutional Review Board of The First Affiliated Hospital, Department of Medical Oncology, of Zhejiang Chinese Medical University, Hangzhou, P. R. China. Only randomized controlled trials (RCTs) were eligible for this review; blinding, language, published or not, duration of treatment, and the duration of follow-up were not considered. The RCT is often considered the gold standard for a clinical trial while are often used to test the efficacy or effectiveness of various types of medical intervention and may provide information about adverse effects, such as Drug reaction [15].

\subsection{Random Assignment}

TKIs Kanglaite Inj or EGFR-TKIs at a ratio of 1:1. The minimization method was implemented via central randomization on the Internet by a clinical research organization (CRO) (Zhejiang Clinical Research Center, Zhejiang, China); the patients were stratified by sex (male vs. female), age (<65 vs. $\geq 65$ ), ECOG PS ( 0 vs. 1 vs. 2 ), staging
(IIIa vs. IIIb vs. IV), smoking status (yes vs. no), first or second line, EGFR mutation status (19 Del vs. 21L858R vs. other rare mutations), TKIs (gefitinib vs. erlotinib vs. icotinib), and by which center the patient was recruited from. During the study, clinicians and subjects were blinded to the type of treatment being given.

\section{Methods}

\subsection{Study Eligibility and Identification}

All randomized trials of CHM plus EGFR-TKIs (gefitinib vs. erlotinib vs. Icotinib) vs the same EGFR-TKIs (gefitinib vs. erlotinib vs. Icotinib) alone, and EGFR-TKIs monotherapy vs placebo or best supportive care were eligible for inclusion. Trials were identified from previous meta-analyses. We electronically searched the Medline, Chinese National Knowledge Infrastructure (Chinese language, English 2010-2019), Pub Med, Cochrane Central Register of Controlled Trails from database inception, CNKI, web of science Wang Fang, and manually searched Chinese-language oncology journals to identify randomized controlled trials (RCTs) of Kanglaite Injection Plus First Line EGFR-TKIs Versus First Line EGFR- TKIs Alone, regardless of their having been published or not, blinding, duration of treatment, or duration of follow-up. The quality of the included trials was assessed using the method recommended by The Cochrane Collaboration (CC). If heterogeneity existed among subgroups, then overall results (OS) were calculated based on a random-effects model; otherwise, a fixed effects model was used. [16]. Therefore, 70 cases of Non-Small Cell Lungs Cancer among Elderly Patients above 60 years of age were selected and randomly divided into two groups: treatment group and control group. Each patient will receive the 
treatment relevant to their group, respectively. Treatment group (expermental) contained 49 cases and control group 21 cases. The treatment group was given the Kanglaite KLT injection as a Chinese herbal Medicine plus first generation Egfr Tkis (gefitinib, erlotinib, Icotinib) and control group was given the first generation Egfr Tkis (gefitinib, erlotinib, Icotinib) drugs alone.

So furthermore, measurements will be included outcomes...While the articles were searching without restriction to language (English, Chinese) or year of publication from 2006 to 2019. In fact reviews are defined as systematic if they included an explicit and repeatable methods section describing the search strategy and explicit inclusion/exclusion criteria.

Cochrane collaboration.

1) Cochrane Reviews $(>4,000)$ registered.

2) Identify, appraise and synthesize research-based evidence and present it in accessible.

3) Format; regularly updated.

4) Focus on interventions.

5) Outstanding general resource.

\subsection{Data Sources}

Web of Science, We also searched the databases of clinical trials such as Current Controlled Trials (http: //www. controlled-trial.com).

\subsection{Study Selection}

(Inclusion and exclusion criteria)

These types of study review will be confined to randomized controlled trials (RCTs) comparing Chinese herbal plus first generation Egfr Tkis to first generation Egfr Tkis alone. If the trial states the "randomization" phase, it will be deemed a randomized study, and the blinding will not be restricted.

\subsection{Inclusion Criteria}

1. Age from 18 to 75 -years-older.

2. Patients were eligible for the study if they had primary NSCLC that was confirmed cytologically, pathologically, or by computed tomography.

3. With histologically or cytologically confirmed stage IIIB or IV NSCLC with histologic features of adenocarcinoma.

4. Patients with a Karnofsky Performance Status (KPS) scores70 and an expected survival time. Finally, patients not treated with chemotherapy or radiotherapy.

5. Harboring activating EGFR mutations (either exon 19 deletion or L858R in exon 21).

6. Eastern Cooperative Oncology Group (ECOG) performance status score 0 to 2 .

7. RCTs.

8. No previous chemotherapy or biologic or immunologic therapy.

9. Measurable lesion according to response Evaluation Criteria in Solid Tumors (RECIST).

\subsection{Exclusion criteria}

Participants meetings one or more of the following criteria will be excluded:

i. Not RCTs;

ii. they have a medical history of cardiovascular disease,

iii. not Rcts,

iv. Study with no EGFR mutation analysis,

v. Due to duplicated publication,

vi. Severe hypersensitivity to gefitinib or any component of CHM,

vii. Active double cancer,

viii. Currently pregnant or breastfeeding,

ix. Patient had liver, brain, or bone metastasis (although asymptomatic patients were eligible),

x. Patients who had just undergone surgery or radiotherapy treatment were also excluded.

\subsection{Interventions}

The trial groups which Patients in the treatment group will be received Kanglaite (KLT) injection as a Chinese herbal Medicine plus first generation Egfr Tkis (gefitinib $250 \mathrm{mg}$ capsules, erlotinib $150 \mathrm{mg}$ capsules, Icotinib $250 \mathrm{mg}$ capsules) and the control groups received first generation Egfr Tkis (gefitinib $250 \mathrm{mg}$ capsules, erlotinib $150 \mathrm{mg}$ capsules, Icotinib $250 \mathrm{mg}$ capsules) alone, regardless of the duration of treatment or follow-up. The basic treatment in both trial and control groups was identical except for KiT injection.(Clinical Trials; CENTRAL), MEDLINE being used the strategy of Dickersin and Larson for controlled clinical trials and EMBASE, CNKI, Pub med. (Excepta Data base) until November 2019.

\section{Outcome Measures}

The primary endpoint was PFS, which was measured with the date of the videography from a random assignment to the date of objective progression or death by the researcher. In addition, the primary Outcome is same as PFS, which is being assessed from the date of randomization to the earliest sign of disease progression, as determined by Computed tomography or magnetic resonance imaging according to the RECIST criteria [17]. The secondary endpoints included a comparison of OS, ORR, DCR, quality of life (QoL), and safety. In fact, the secondary Outcomes are also MST, objective response rate, disease control rate and safety. MST will be assessed from the date of randomization until $50 \%$ of participants are dead from any cause, with the use of methods that are similar to those used for the analysis of PFS. Tumor response will be assessed every six weeks during the first year after randomization, and every two months after the first year, until disease progression. The follow-up time of both groups is two years after recruitment.

In addition, Computed tomography or magnetic resonance imaging was used to assess tumor at baseline and every 8 weeks until disease progression. Patients who received more than $80 \%$ of the expected dose of EGFR-TKI and CHM were 
considered adherent. We recorded treatment-emergent AEs (TEAEs) per the Medical Dictionary for Regulatory Activities (version 15.0) and graded them using the National Cancer
Institute's common terminology criteria for adverse events (version 3.0).

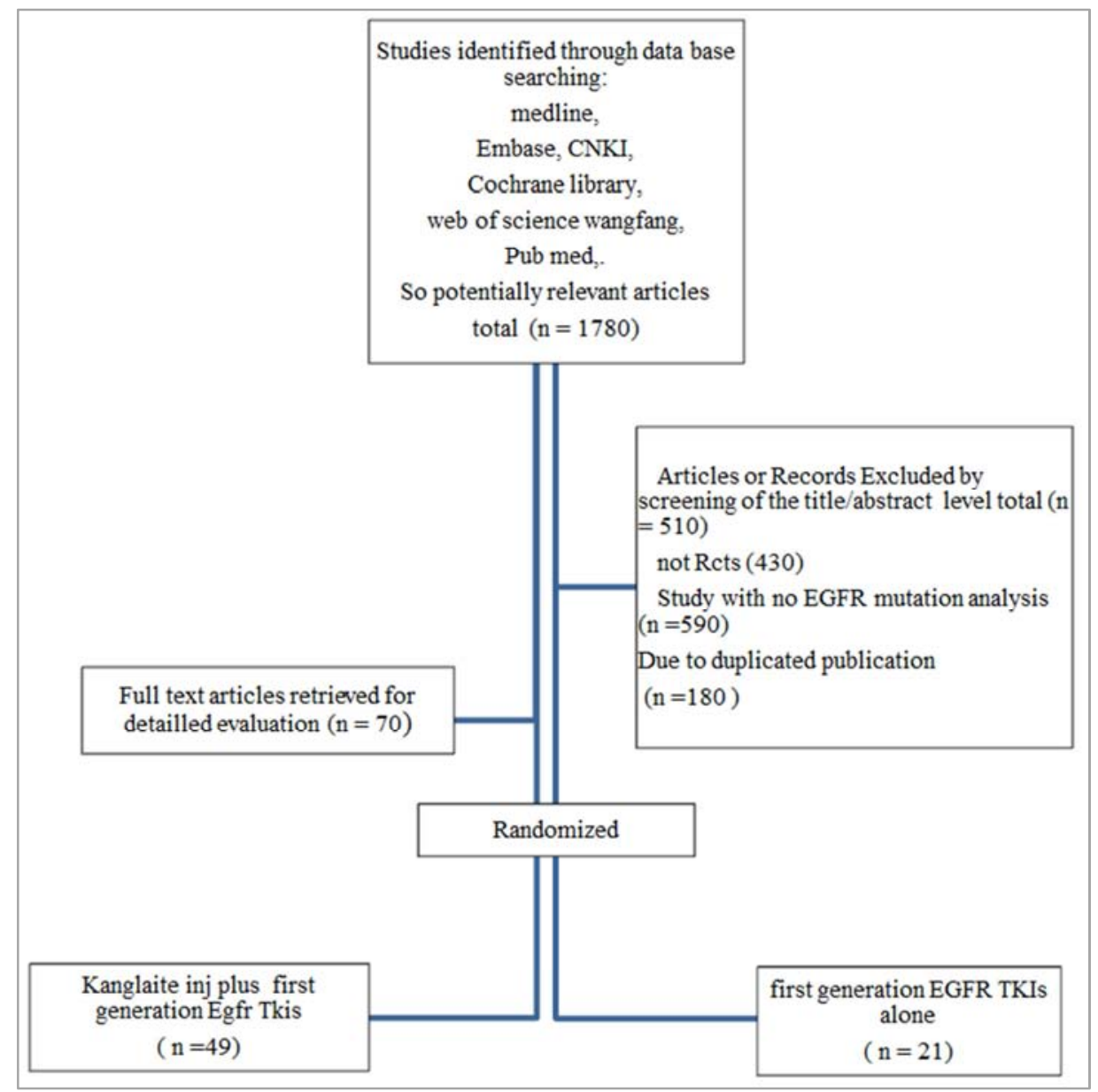

Figure 5. CONSORT diagram: trial profile at the cut-off date for analysis (January 5, 2019) P D, progressive disease.

QoL was collected using the Functional Assessment of Cancer Therapy-Lung (FACT-L) questionnaire, 2002; Thongprasert et al., 2011) and the Lung Cancer Symptom Scale (LCSS).

\subsection{Quality-of-Life}

Improvement Quality of life before and after treatment was assessed using the KPS. Only data for patients whose KPS scores improved by less than 10 points (the minimal clinically significant difference) after treatment were extracted.

\subsection{Symptom Improvement}

The percentage of patients exhibiting improvement in the symptoms of cough, hemoptysis, chest pain, fever, fatigue, and anorexia was assessed. We also calculated the percentage of patients whose symptoms resolved completely. Improvement in symptoms was assessed according to the information provided in each included study. Specifically, for the pooled analysis of the trials in which symptom improvement was reported, symptoms were scored according to their degree of severity (ie, grades 1III). For the symptoms of cough, chest pain, and dyspnea, grade I was assigned when the symptoms did not influence daily life, and grade III was assigned when the symptoms were severe, with a marked influence on daily life; symptoms between grade I and III were assigned grade II. For hemoptysis, sputum with blood was assigned grade I, sputum with blood clots or sputum with $\sim 10 \mathrm{mUd}$ of blood was assigned grade II, and extremely bloody sputum or sputum with $>10 \mathrm{mUd}$ of blood was assigned grade III.

\subsection{Adverse Events}

Adverse events (AEs) were evaluated at the completion of treatment and included bone marrow suppression (leukopenia, anemia, and thrombocytopenia), nausea and vomiting, phlebitis, hepatic dysfunction, and renal dysfunction. According to the WHO grading criteria for acute and subacute toxicity of anticancer drugs, we only calculated AEs of grades II to IV. If patients withdrew from the study due to an AE, we also included these AEs.

\subsection{Quality Assessment}

The quality of the trials was assessed according to the 
Cochrane Collaboration's criteria11: (1) minimization of selection bias (ie, were the randomization procedure and the allocation concealment adequate); (2) minimization of performance bias (ie, were the patients who received treatment and people who administered the treatment blinded to the interventions); (3) minimization of attrition bias (ie, were withdrawals and dropouts completely described and was the analysis based on intent to treat [ITT $\}$ ); and (4) minimization of detection bias (ie, were outcome assessors blinded to the interventions). Based on these criteria, the studies were broadly subdivided into the following 3 categories: $A=$ all quality criteria met, low risk of bias; $\mathrm{B}=$ greater than 1 of the quality criteria only partially met, moderate risk of bias; and $\mathrm{C}=$ greater than 1 criteria not met, high risk of bias.

Each trial was assessed independently by one author (Wang) and was checked by another author (Shu). Differences were resolved by discussion.

\subsection{Data Extraction}

Data from each included trial were extracted independently by one author (David et al.) and checked by correspondence author (Shu) using a standard extraction form. The form included the following items:

1) General information: published/unpublished; language; authors; article; journal title, year, volume, issue, and page numbers; and funding source;

2) Trial design: predetermined sample size, generation of randomization sequence, allocation concealment method, blinding of information, statistical methods, and attrition;

3) Participants: diagnostic criteria, total number of patients and number of patients in the comparison groups, baseline characteristics (eg, age, gender), inclusion criteria, exclusion criteria, and study settings;

4) Intervention: type of chemotherapy regimen, duration, time, and dose; co-intervention; control; withdrawals, dropouts, and lost to follow-up; and

5) Outcome: outcomes at the end of treatment. The number and type of AEs were also extracted. If the aforementioned data were not available in the trial report, further information was sought by corresponding with the original principal investigator.

\subsection{Statistical Analysis}

Data were analyzed using Meta View 4.2.8 in Review Manager 4.2 (Cochrane collaboration, Oxford, United Kingdom). Meta-analysis was conducted by pooling the different drugs regimens combined together KLT injection plus First Generation Egfr Tkis and comparing these with first generation Egfr tkis regimens alone for an overall analysis; however, the analysis was divided according to subgroups that were formed based on KLT Injection Plus First Generation Egfr Tkis regimens. Sensitivity analyses were conducted by excluding low quality trials (David et al.). Analyses were conducted using the ITT principle when possible. Relative risk (RR) was used to analyze dichotomous data. If heterogeneity existed among subgroups, then overall results OR were calculated based on the random effect model; otherwise, the fixed effect model was used [22]. The random effect model was also used to check whether its use might change the direction of the results in cases where heterogeneity was not tested. Heterogeneity was tested using the $\mathrm{Z}$ score and $\mathrm{X}^{2}$, and $\mathrm{P}<0.1$ was considered statistically significant.

\section{Results}

In summary, Electronic database searches yielded 1780 citations, 70 cases retrieved for detail evaluation of Non-Small Cell Lungs Cancer among Elderly Patients above 70 years of age were selected and randomly divided into two groups: treatment group and control group. Each patient received the treatment relevant to their group, respectively. Treatment groups (expermental) contained 49 cases and control group 21 cases. So therefore, Kanglaite Injection plus Egfr Tkis (Erlotinib, Gefitinib, Afatinib, Dacomitinib, and Osimertinib) was effective than plus Egfr Tkis (Erlotinib, Gefitinib, Afatinib, Dacomitinib, and Osimertinib) alone $(\mathrm{P}<0.05)$, the $\mathrm{I}^{2}=99 \%$ in Mean difference IV Fixed CI 95\% See Figure 3. Comparison on clinical efficacy between the two groups, comparison on total effective rate between the two groups showed that data from treatment group was apparently better than those from control group with significant statistical differences $(\mathrm{P}<0.05)$, the $\mathrm{I}^{2}=99 \%$ in Mean difference IV Fixed CI 95\% see Table 3, [18].

Table 4. Clinical profile of Patients with PFS of EGFR-TKIs treatment $>6$ months.

\begin{tabular}{|c|c|c|c|c|c|c|c|c|c|c|c|c|}
\hline \multirow{3}{*}{ Case } & \multirow{3}{*}{ Sex } & \multicolumn{2}{|l|}{ Age } & \multirow{3}{*}{ Stage } & \multirow{3}{*}{ Smoking } & \multirow{3}{*}{ Gene type } & \multirow{3}{*}{ Sex } & \multirow{2}{*}{\multicolumn{2}{|c|}{$\begin{array}{l}\text { Age } \\
\text { (Years) }\end{array}$}} & \multirow{3}{*}{ Stage } & \multirow{3}{*}{ Smoking } & \multirow{3}{*}{ Gene type } \\
\hline & & \multicolumn{2}{|c|}{ (Years) } & & & & & & & & & \\
\hline & & Mean & SD & & & & & Mean & SD & & & \\
\hline 01 & $\mathrm{M}$ & 66 & 18.1 & IIIB & No & T790M+19delete & $\mathrm{F}$ & 59 & 18.3 & IV & No & S7681 \\
\hline 02 & $\mathrm{M}$ & 62 & 17.03 & IV & No & 20 insertion+19del & $\mathrm{F}$ & 56 & 17.4 & IV & No & $\mathrm{T} 790 \mathrm{M}+\mathrm{L} 858 \mathrm{R}$ \\
\hline 03 & M & 64 & 17.5 & IV & Yes & 20insertion & $\mathrm{F}$ & 49 & 15.2 & IV & No & S7681+L858R \\
\hline 04 & $\mathrm{M}$ & 47 & 12.9 & IV & Yes & 20insertion+L858R & $\mathrm{F}$ & 63 & 19.6 & IV & No & T790M+G719X \\
\hline 05 & M & 68 & 18.6 & IV & No & T790M+L858R & $\mathrm{F}$ & 55 & 17.1 & IV & No & S76881+19delete \\
\hline Total $\%$ & & 364 & $99.73 \%$ & & & & & 321 & $99.7 \%$ & & & \\
\hline
\end{tabular}

Clinical profile of Patients with PFS of EGFR-TKIs treatment $>6$ months were divided into two groups according to their Gender, Age, Stage \& Gene type. In summary, the sex male were affected 364 in total $99.73 \%$ than the Female 321 in total $99.7 \%$ in different gene types. HR, hazard ratio; ITT, intent to treat; ORR, overall response rate; OS, over survival; PFS, progression-free survival; CR, complete response; PR, partial response; SD, stable disease; PD, progressive disease; DCR, disease control rate; DoR, duration of response. A two-sided P-value was derived from log-rank test for PFS and from Fisher's exact test for tumor response. 


\section{IPASS: PFS by EGFR Mutation Status}

- Randomized phase III trial; previously untreated pts $(\mathrm{N}=1217)$

- PFS: Gefitinib superior to carboplatin/paclitaxel in ITT population

- EGFR mutations strongly predicted PFS (and tumor response) to first-line gefitinib vs carboplatin/paclitaxel

EGFR Mutation-Positive

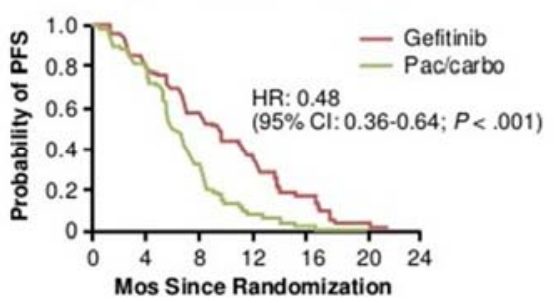

EGFR Mutation-Negative

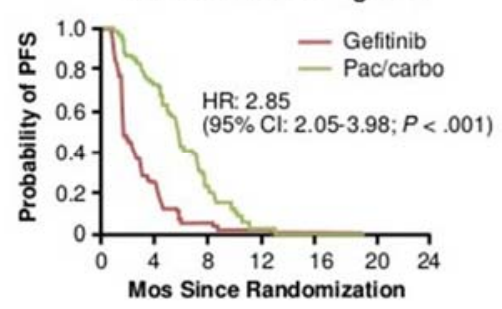

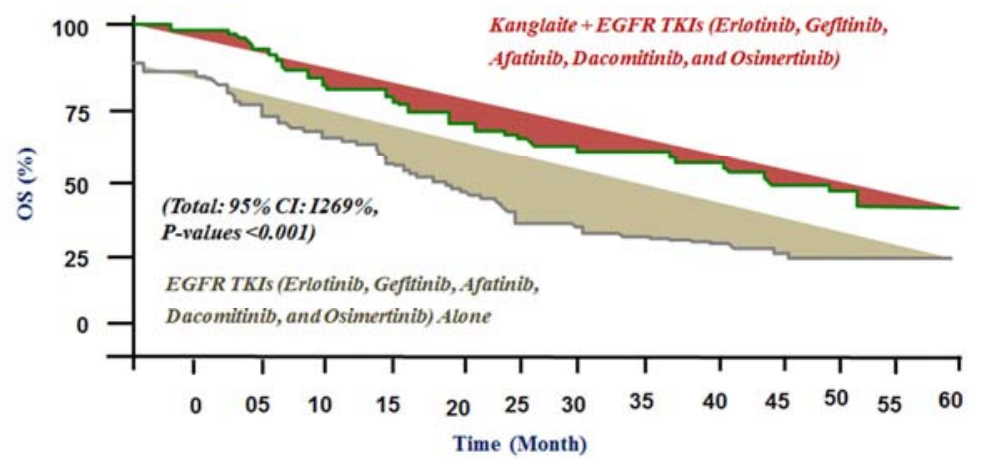

Figure 6. Kanglaite + EGFR TKIs (Erlotinib, Gefitinib, Afatinib, Dacomitinib, and Osimertinib) is significantly benefit than EGFR TKIs Alone.

Table 5. Comparison on Karnofsky and score between two Groups the experimental group and control group.

\begin{tabular}{|c|c|c|c|c|c|c|c|c|}
\hline \multirow[t]{3}{*}{ Authors Names } & \multirow[t]{3}{*}{ Year } & \multicolumn{3}{|c|}{ Group I } & \multicolumn{4}{|c|}{ Group I } \\
\hline & & \multicolumn{3}{|c|}{ Experimental Group } & \multicolumn{4}{|c|}{ Control Group } \\
\hline & & Mean & SD & Total & Mean & SD & Total & $\%$ \\
\hline Ailing Cao & 2017 & 65.5 & 5.1 & 70 & 25.6 & 20.1 & 45 & $5.3 \%$ \\
\hline Haoram Zhai & 2014 & 55.6 & 8.9 & 64 & 24.8 & 19.8 & 79.8 & $4.8 \%$ \\
\hline Sarah Burdett & 2006 & 28.8 & 3.6 & 32 & 25.5 & 20.4 & 45.9 & $37.5 \%$ \\
\hline Total $\%$ & - & 149.9 & 17.2 & 300 & 75.9 & 60.3 & 170.7 & $47.6 \%$ Total: $95 \%$ CI P-values \\
\hline
\end{tabular}

a. All HRs and corresponding P-values were unadjusted, except as otherwise noted. HR values for TKI+CHM and TKI were derived from a Cox regression analysis, and one-and two-sided P-values were derived from the Wald test from the Cox model. c Adjusted for EGFR mutation type, age, sex, smoking status, ECOG performance status, stage, TKI drugs, TCM syndrome, and prior chemotherapy therapy. $d$ Defined as the time from random assignment to the first date of disease progression. e Defined as the time from random assignment to the date of death from any cause. f Percentages may not add up to $100 \%$ because of rounding. $g$ Defined as the time from the date of the first CR or PR to the first date of progressive disease (per RECIST version 1.1). h Analyzed in the ITT population with CR or PR.

\begin{tabular}{|c|c|c|c|c|c|c|c|c|}
\hline \multirow[b]{2}{*}{ Study or Subgroup } & \multicolumn{2}{|c|}{ Kanglaite Inj + EGFRTKIs } & \multicolumn{2}{|c|}{ EGFR TKIs Alone } & \multirow[b]{2}{*}{ Weight } & \multirow{2}{*}{$\begin{array}{c}\text { Risk Ratio } \\
\text { M-H, Fixed, } 95 \% \mathrm{Cl}\end{array}$} & \multirow{2}{*}{\multicolumn{2}{|c|}{$\begin{array}{c}\text { Risk Ratio } \\
\text { M-H, Fixed, } 95 \% \mathrm{Cl}\end{array}$}} \\
\hline & Events & Total & Events & Total & & & & \\
\hline Aling Cao 2017 & 24 & 324 & 17 & 288 & $5.3 \%$ & $1.25[0.69,2.29]$ & & \\
\hline Hacran Zhai 2014 & 26 & 90 & 62 & 96 & $17.8 \%$ & $0.45[0.31,0.64]$ & & \\
\hline Jimwu Peng 2017 & 40 & 60 & 45 & 65 & $12.8 \%$ & $0.96[0.76,1.23]$ & & \\
\hline Liu Pu 2019 & 45 & 56 & 47 & 56 & $14.0 \%$ & $0.96[0.81,1.14]$ & & - \\
\hline Sarah Burdett 2006 & 72 & 84 & 78 & 85 & $23.0 \%$ & $0.93[0.84,1.04]$ & & \\
\hline Xuemei Liu 2008 & 48 & 69 & 70 & 89 & $18.1 \%$ & $0.88[0.73,1.07]$ & & - \\
\hline Yang Zhou 2008 & 20 & 30 & 50 & 70 & $8.9 \%$ & $0.93[0.70,1.25]$ & & \\
\hline Total $(95 \% \mathrm{Cl})$ & & 713 & & 749 & $100.0 \%$ & $0.86[0.79,0.94]$ & & \\
\hline Total events & 275 & & 369 & & & & & \\
\hline \multicolumn{7}{|c|}{$\begin{array}{l}\text { Heterogeneity, } C h i^{2}=19.18, d f=6(P=0.004) ; I^{2}=69 \% \\
\text { Test for overall effect: } Z=3.26(P=0.001)\end{array}$} & $0.5 \quad 0.7$ & $11.5 \quad 2$ \\
\hline
\end{tabular}

Figure 7. Forest plot of comparison: outcome: Progression Free Survival (PFS). Kanglaite Injection Plus EGFR TKIs (Erlotinib, Gefitinib, Afatinib, Dacomitinib, and Osimertinib) Versus EGFR TKIs (Erlotinib, Gefitinib, Afatinib, Dacomitinib) Alone Risk Ratio (RR, 95\% CI). 


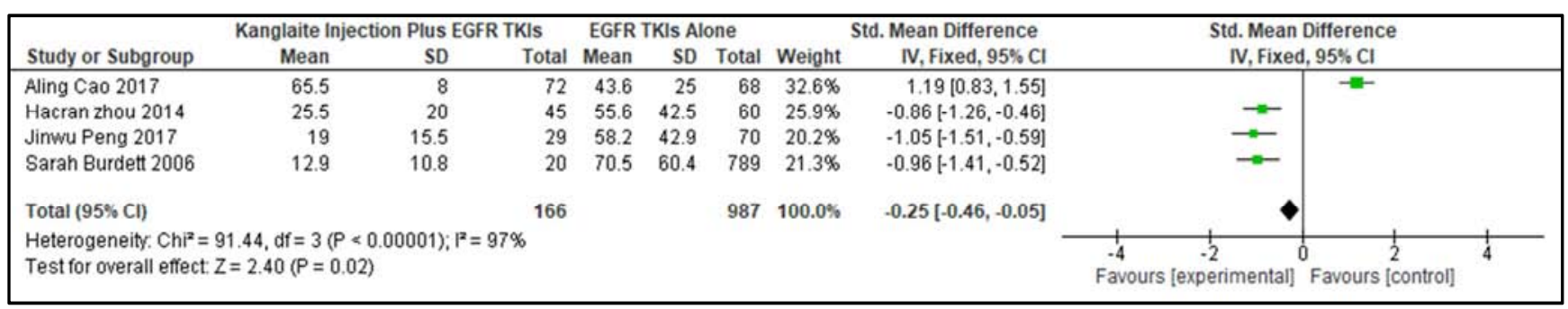

Figure 8. Forest plot of comparison: outcome: Quality of Life (Qol). Kanglaite Injection Plus First-Line Epidermal Growth Factor Receptor (EGFR) Tyrosine Kinase Inhibitors (TKIs) Versus First-Line EGFRTKIs Alone. Std. Mean Different, 95\% CI).

\begin{tabular}{|c|c|c|c|c|c|c|c|c|c|}
\hline \multirow[b]{2}{*}{ Study or Subgroup } & \multicolumn{2}{|c|}{ Kanglaite Inj + EGFR TKIs } & \multicolumn{2}{|c|}{ EGFR TKIs } & \multirow[b]{2}{*}{ Weight } & \multirow{2}{*}{$\begin{array}{c}\text { Risk Ratio } \\
\text { M-H, Fixed, } 95 \% \mathrm{Cl}\end{array}$} & \multirow{2}{*}{\multicolumn{3}{|c|}{$\begin{array}{c}\text { Risk Ratio } \\
\text { M-H, Fixed, } 95 \% \mathrm{Cl}\end{array}$}} \\
\hline & Events & Total & Events & Total & & & & & \\
\hline Sarah Burdet 2006 & 5 & 30 & 10 & 35 & $22.3 \%$ & $0.58[0.22,1.52]$ & 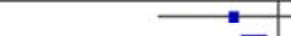 & E & \\
\hline Xuemei Liu 2008 & 20 & 40 & 30 & 45 & $68.1 \%$ & $0.75[0.52,1.09]$ & & & \\
\hline Yang Zhou 2008 & 4 & 10 & 5 & 15 & $9.6 \%$ & $1.20[0.42,3.41]$ & & & \\
\hline Total $(95 \% \mathrm{Cl})$ & & 80 & & 95 & $100.0 \%$ & $0.76[0.54,1.06]$ & & & \\
\hline Total events & 29 & & 45 & & & & & & \\
\hline $\begin{array}{l}\text { Heterogeneity. } \mathrm{Chi}^{2}= \\
\text { Test for overall effect }\end{array}$ & $\begin{array}{l}\text { 1.04, df }=2(P= \\
Z=1.62(P=0\end{array}$ & $P^{2}=0 \%$ & & & & & $\begin{array}{ll}0.05 & 0.2 \\
\text { Favours [experimental] }\end{array}$ & $\begin{array}{c}5 \\
\text { Favours [control] }\end{array}$ & 20 \\
\hline
\end{tabular}

Figure 9. Forest plot of comparison: outcome: Objective Response Rate (ORR). Kanglaite Plus EGFR TKKIs (Erlotinib, Gefitinib, Afatinib, Dacomitinib, and Osimertinib) Versus EGFR TKIs (Erlotinib, Gefitinib, Afatinib, Dacomitinib) Alone. Std. Mean Difference (Risk Ratio).

\begin{tabular}{|c|c|c|c|c|c|c|c|c|c|}
\hline \multirow[b]{2}{*}{ Study or Subgroup } & \multicolumn{2}{|c|}{ Kanglaite Inj + EGFR TKIs } & \multicolumn{2}{|c|}{ EGFR TKIs } & \multirow[b]{2}{*}{ Weight } & \multirow{2}{*}{$\begin{array}{c}\text { Odds Ratio } \\
\text { M-H, Fixed, } 95 \% \mathrm{Cl}\end{array}$} & \multirow{2}{*}{\multicolumn{3}{|c|}{$\begin{array}{c}\text { Odds Ratio } \\
\text { M-H, Fixed, } 95 \% \mathrm{Cl}\end{array}$}} \\
\hline & Events & Total & Events & Total & & & & & \\
\hline Jinwu Peng 2017 & 27 & 90 & 62 & 90 & $69.9 \%$ & $0.19[0.10,0.36]$ & 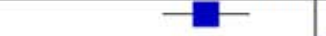 & & \\
\hline Liu Pu 2019 & 24 & 360 & 20 & 360 & $30.1 \%$ & $1.21[0.66,2.24]$ & - & $=-$ & \\
\hline Total $(95 \% \mathrm{Cl})$ & & 450 & & 450 & $100.0 \%$ & $0.50[0.33,0.76]$ & & & \\
\hline Total events & 51 & & 82 & & & & & & \\
\hline $\begin{array}{l}\text { Heterogeneity. } \mathrm{Chi}^{2}= \\
\text { Test for overall effect }\end{array}$ & $\begin{array}{l}16.67, d f=1(f \\
Z=3.25(P=0\end{array}$ & 1); $; P^{2}=$ & $94 \%$ & & & & 0.010 .1 & $\begin{array}{c}10 \\
\text { Favours [control] }\end{array}$ & 100 \\
\hline
\end{tabular}

Figure 10. Forest plot of comparison: outcome: disease control rate (DCR). Kanglaite Plus EGFR TKKIs (Erlotinib, Gefitinib, Afatinib, Dacomitinib, and Osimertinib) Versus EGFR TKIs (Erlotinib, Gefitinib, Afatinib, Dacomitinib, and Osimertinib) Alone. Odds Ratio (OR) I2=94\%, P<0.001.
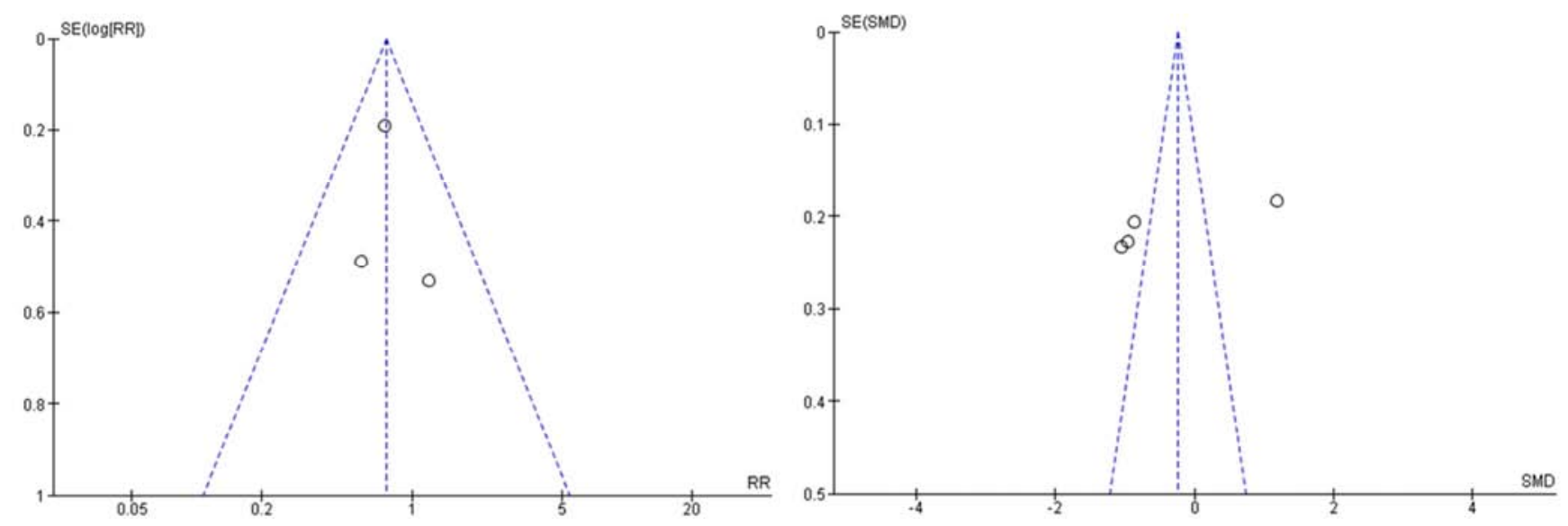

Figure 11. Analysis Funnel plot of comparison: outcome: Progression Free Survival (PFS). Kanglaite Injection Plus EGFR TKIs (Erlotinib, Gefitinib, Afatinib, Dacomitinib, and Osimertinib) Versus EGFR TKI Gefitinib, Afatinib, Dacomitinib, and Osimertinib) Alone. Std. Mean Difference (SMD).

\section{Conclusion}

To the best of our knowledge, to examine the efficacy and safety of first- and second-line TKIs+Kanglaite Injection versus EGFR-TKIs alone in patients with advanced adenocarcinoma whose tumors harbor EGFR activating mutations. EGFR-TKIs have proved effective in first- or second-line therapy for advanced NSCLC First-line treatment. EGFR mutation is the best established, oncogenic target for management of advanced stage NSCLC. The predictive power of EGFR mutation is confirmed in multiple randomized phase 3 studies comparing first- (gefitinib or erlotinib i.e. reversible inhibitors) or second-generation (Afatinib i.e. irreversible inhibitors) EGFR-TKIs with Kanglaite Injection. The 
improvement in response rate (ORR) and PFS is consistent across all age groups, genders, smoking status and performance status (PS). Notably, none of the above studies have shown any benefit in OS for an EGFR-TKI with Kanglaite Injection, likely due to the high level of crossover. EGFR-TKIs represent the standard of care as first-line treatment for advanced EGFR-mutated NSCLC. Patients with PS 3-4 may also be offered an EGFR-TKI as they are likely to receive a similar clinical benefit as patients with good PS. Patients who have benefited from EGFR-TKI treatment may continue to receive the same therapy beyond initial radiological progression as long as they are clinically stable. The chance to go beyond progression with EGFR-TKI can be considered in patients with oligo- progressive disease, with a consolidation of the progressing lesions with radiation therapy, resulting in a longer PFS. Patients with localized distant progression and ongoing systemic control, continuation of treatment with EGFR-TKI in combination with local treatment of progressing metastatic sites may be considered. A meta-analysis of randomized trials of treatment-native patients reported that EGFR-TKIs statistically significantly prolonged PFS overall, but because of the high rate of crossover at progression, EGFR-TKI had a shorter OS than those who were randomly assigned to chemotherapy (12.8 months, 95\% CI: $11.4-14.3$ vs. 19.8 months, 95\% CI: 17.6 21.7) (Lee et al., 2017). EGFR T790M is a mutation associated with acquired resistance to EGFR-TKI therapy and has been reported in approximately $60 \%$ of patients with disease progression after the initial treatment with erlotinib and gefitinib (Yu et al., 2013; Yu et al., 2015). Efforts have been made to delay resistance to EGFR-TKI. Osimertinib has shown to the potential to prolong PFS to 22.1 months in treatment-naive patients with EGFR advanced NSCLC. However, these strategies overdraft subsequent chemotherapy and targeted therapy and might not benefit OS. CHM treatment could be given according to the patients' physical status, syndrome differentiation, and type of cancer treatment. Patients with lung cancer are receiving the theoretical directions of "treatment of cancer by strengthening antipathogenic ability." In China, CHM as an adjuvant therapy has shown the potential to reduce EGFR TKIs, chemotherapy toxicity, prolong survival rate, enhance immediate tumor response, and improve Karnofsky performance status (KPS) in advanced NSCLC patients, but its efficacy remains largely unexplored (Li et al., 2013).

Kanglaite Inj as CHM combined with EGFR-TKI has been demonstrated to be effective in both retrospective and small sample prospective clinical trials (Yang et al., 2014; Hung et al., 2017). The results of the study conclusively show that EGFR TKIs plus CHM as a first- or second-line therapy provides significantly prolonged PFS and ORR compared with EGFR TKIs alone in ADC with EGFR activating mutations. Condition. The results of this study showed that the total treatment effective rate of the experimental group which using Kanglaite plus EGFR TKIs plus CHM was significantly higher than that of the control group (Chemotherapy drugs alone) $(\mathrm{P}<0.05)$, the $\mathrm{I} 2=99 \%$ in Mean difference IV Fixed CI
95\%. The incidence of adverse reactions in patients of the experimental group was lower than that of the control group and the differences between groups were $\mathrm{P}<0.05$, with statistically significant. In addition, the study with immune indicators $(\mathrm{CD} 3+, \mathrm{CD} 4+, \operatorname{IgG}$, and $\operatorname{IgA})$ were better than that of the control group, the differences between the groups were $\mathrm{P}<0.05$, have statistically significant, and indicate that the drug can enhance the immune function of cancer EGFR TKIs patients. In the EGFR TKIs of advanced NSCLC, the addition of Kanglaite injection can improve the effect of EGFR TKIs, reduce the side effects of EGFR TKIs, and also significantly improve the immune function of patients.

\section{Abbreviation}

EGFR: epidermal growth factor receptor

TKIs): Tyrosine Kinase Inhibitors

KPS: Karnofsky performance status

PFS: Progression Free Survival

PD: Progression disease

LN: lymph node

SD: Stable disease

PR: Partial response

LAR: long-acting release

CM: Chemotherapy

TCM: Traditional Chinese Medicine

WM: Western Medicine

KGLI: Kanglaite Injection

NSCLC: Non-Small Cell Lung Cancer

\section{Data Availability Statement}

The datasets generated for this study are available on request to the corresponding author.

\section{Ethics Statement}

This study was carried out in accordance with the recommendations of NCCN Clinical Practice Guidelines Oncology, Institutional Review Board of Department of Medical Oncology, The First Affiliated Hospital of Zhejiang Chinese Medical University with written informed consent from all subjects. The First Clinical Medical College of Medicine, Zhejiang Chinese Medical University (International Education College IEC), Hang Zhou P. R. China.

\section{Author Contributions}

Conception and design: Shu Qijin, David Kayembe and Financial support: Wenpei Zhu, Ying Wang (ZCMU IEC); Administrative support: Wenpei Zhu, Ying Wang; Provision of study materials or patients: Chen Huihui and; Collection and assembly of data: Liang Yi, David Kayembe, Wang Dan and Data analysis and interpretation: Wang Dan, Zhang Gaochenxi, Zhu Junjing, David Kayembe. Manuscript writing: All authors; Final approval of manuscript: All authors; 
Accountable for all aspects of the work: All authors.

\section{Funding}

This study was sponsored by the Zhejiang Chinese Medical University (International Education College IEC), Hangzhou, Zhejiang 310053; P. R. China.

\section{Acknowledgements}

We are grateful to Professor Dr Shu Qijin's guidance \& an excellent mentor who was agreed to lead this work. Furthermore, we are greateful and heartfelt thanks to our professor Dr. Wang Ying Dean of ZCMU IEC, to our Advisor Dr Zhu wenpei and thankful to our colleague's experts who were invited to manage the statistical analysis.

\section{References}

[1] Molina JR, Ad jei AA, JettJR. Advances in chemotherapy ofnon-small cell lung cancer. Chest. 2006; 130: 1211-1219.

[2] The American Cancer Society medical and editorial content team.www.cancer.org.

[3] Encun H, Jie C, Lu G, Hang S, Jing D. Systematic evaluation of the efficacy of Kanglaite combined with GP regimenting the treatment of advanced non-small cell lung cancer [J]. Mod On col Med 2015; 7: 960-965.

[4] Yuan W, Shuang H, Min L, Qi F, Zhao L. Clinical study of Kanglaite injection in the treatment of advanced non-small cell lung cancer with gemcitabine combined with cisplatin regimen [J]. Chin J Clin Pharm 2017; 33: 2354-2356, 2360.

[5] Bao Y., Xia L., Jang H. The experiment and study on cellular apoptosis produced by KLT Injections in pancreatic cancer cells. Shanghai Journal Medicine. 2004; 27: 421-424. [Google Scholar].

[6] Schwartzberg L. S., Arena F. P., Bienvenu B. J., et al. A randomized, open-label, safety and exploratory efficacy study of Kanglaite injection (KLTi) plus gemcitabine versus gemcitabine in patients with advanced pancreatic cancer. Journal of Cancer. 2017; 8 (10): 1872-1883. Doi: 10.7150/jca.15407. [PMC free article] [PubMed] [CrossRef] [Google Scholar].
[7] Shi G., Zheng X., Zhang S., et al. Kanglaite inhibits EMT caused by TNF- $\alpha$ via NF- $\kappa$ B inhibition in colorectal cancer cells. Oncotarget. 2017; 9: 6771-6779. Doi: 10.18632/oncotarget.23645. [PMC free article] [PubMed] [CrossRef] [Google Scholar].

[8] Xinna D, Shufang Z, Yang L, Wei MQ, Zhou SF. Therapeutic effect of Kanglaite injection combined with chemotherapy on elderly patients with advanced non-small cell lung cancer [J]. Mod J Integr Tradit Chin West Med 2015; 18: 1951-1953, 1957.

[9] Hongxue Z, Chunsheng Z, Lianbin L, Galluzzi L, Senovilla L. Effects of Kanglaite combined with gefitinib on immune function and quality of life in patients with advanced non-small cell lung cancer $[\mathrm{J}]$. Chin J Pract Diagn Ther 2014; 28: 930-931.

[10] Ting J, Qi P. Evaluation of efficacy and safety of Kanglaite combined with chemotherapy versus chemotherapy alone in advanced non-small cell lung cancer: Meta-analysis [J]. Int $\mathrm{J}$ Res 2017; 37: 116-123.

[11] Selvin E, Parrinello CM, Sacks DB, Coresh J. Trends in prevalence and control of diabetes in the United States, 19881994 and 1999-2010. Ann Intern Med. 2014; 160: 517-525.

[12] Surveillance, Epidemiology, and End Results (SEER) Program. SEER*Stat Database: Incidence-SEER 13 Regs Research Data with Delay-Adjustment, Malignant Only, Nov. 2017 Sub (1992-2015) xxaaa Katrina/Rita Population Adjustmentxxbbb-Linked To County Attributes-Total US, 1969-2016 Counties. Bethesda, MD: National Cancer Institute, Division of Cancer Control and Population Sciences, Surveillance Research Program; 2017.

[13] Jun Y, Xiao S. Clinical analysis of gemcitabine and cisplatin combined with Kanglaite injection in the treatment of advanced non-small cell lung cancer [J]. J Clin Exp Med 2017; 16: 1195-1198.

[14] Yinzhong Q. The effect of Kanglaite combined with radiotherapy on non-small cell lung cancer and immune function of patients [J]. Pract J Cancer 2016; 31: 578-580.

[15] Meng H, Shubo C, Qing S, Xu J. Efficacy and safety of GP regimen combined with Kanglaite injection in the treatment of advanced non-small cell lung cancer [J]. Pract J Cancer 2017; 32: $289-291$.

[16] Mok TS, Wu YL, Thongprasert S, et al. Gefitinib or carboplatin-paclitaxel in pulmonary adenocarcinoma. N Engl J Med. 2009; 361: 947-957. 